\title{
Unique identification of a multi-layered fluid-solid medium
}

\author{
Yanli Cui ${ }^{1}$, Xiliang $\mathrm{Li}^{2}$ and Fenglong Qu ${ }^{1 *}$ (1)
}

"Correspondence:

fenglongqu@amss.ac.cn

'School of Mathematics and Information Sciences, Yantai

University, Yantai, P.R. China Full list of author information is

available at the end of the article

\section{Springer}

\begin{abstract}
This paper is concerned with the inverse scattering of time-harmonic acoustic plane waves by a multi-layered fluid-solid medium in the three dimensional space. We establish the global uniqueness in identifying the embedded penetrable solid obstacle, the surrounding fluid medium and its wave number from the acoustic far-field pattern for all incident plane waves at a fixed frequency. The proof depends on constructing different kinds of interior transmission problems in appropriate small domains and the a priori estimates derived for both the elastic wave fields in the embedded solid obstacle and the acoustic wave fields in the surrounding fluid medium.
\end{abstract}

Keywords: Inverse scattering; Uniqueness; Fluid-solid interaction; Multi-layered medium

\section{Introduction}

Consider the inverse problem of scattering of time-harmonic acoustic plane waves by a bounded penetrable elastic obstacle embedded in an inhomogeneous acoustic background medium. This gives rise to the fluid-solid interaction problem, which has important applications in lots of fields such as the medical imaging, non-destructive testing and the material science (cf. $[33,38]$ and the references cited therein).

The fluid-solid interaction problem under consideration is modeled by the Helmholtz equation with different wave numbers in the layered fluid medium, where the solution is continuous across the interface, and satisfies the Navier equation in the solid obstacle. A coupled transmission condition is imposed on the interface between the solid obstacle and the surrounding fluid medium. The well-posedness of the forward scattering problem can be established by the coupling method of a variational and boundary integral equation techniques (cf. [13, 20]).

In the current paper, we are interested in the inverse problem of recovering the embedded penetrable solid obstacle, the surrounding fluid medium and the corresponding wave number by means of acoustic far-field data. This is a challenging topic since both the embedded solid obstacle and the surrounded background fluid medium are unknown. For the case when the solid obstacle embedded in a homogeneous background fluid medium, there already exist diverse inversion algorithms of reconstructing the elastic body from

(c) The Author(s) 2020. This article is licensed under a Creative Commons Attribution 4.0 International License, which permits use sharing, adaptation, distribution and reproduction in any medium or format, as long as you give appropriate credit to the original author(s) and the source, provide a link to the Creative Commons licence, and indicate if changes were made. The images or other third party material in this article are included in the article's Creative Commons licence, unless indicated otherwise in a credit line to the material. If material is not included in the article's Creative Commons licence and your intended use is not permitted by statutory regulation or exceeds the permitted use, you will need to obtain permission directly from the copyright holder. To view a copy of this licence, visit http://creativecommons.org/licenses/by/4.0/. 
acoustic measurements; see e.g., the iterative optimization methods in [6, 7], non-iterative qualitative methods in [21, 24, 25, 38], and the integral equation method in [37]. Meanwhile, some uniqueness results have also been established. A well-known uniqueness theorem of identifying a bounded solid body from acoustic far-field data was firstly proved in [27]. The proof in [27] was then simplified by [24] due to Hähner's idea, where a $H^{2}$ regularity of the related solutions was applied. A different technique was proposed in [32] in determining a bounded solid obstacle from acoustic measurements. We also refer to $[1,10]$ for the analysis of the time-dependent fluid-solid interaction problems.

It is well known that the uniqueness issue for the full acoustic inverse medium scattering problems has been extensively studied (cf. [2, 5, 9, 14, 15, 22, 23, 28, 29, 34, 39] and the references cited therein). Most of them are focused on the unique recovery of the shape of a penetrable obstacle in the case when the solution is discontinuous across the penetrable interface. For example, based on the idea of constructing singular solutions of related scattering problems, Isakov [16] established the first uniqueness result for the penetrable obstacles. Hähner [12] proposed a different technique for an inverse anisotropic medium scattering problem. A uniqueness theorem can also be found in [8] for the case when an impenetrable acoustic obstacle embedded in a piecewise homogeneous medium. Relying on constructing complex geometrical optics solutions method, [26] proved a uniqueness theorem in recovering a penetrable obstacle, which was then extended to the case of the elastic scattering problem [19] and Maxwell's equations [18]. The reader is referred to [36] for a novel method in recovering the shape and location of penetrable obstacles for full acoustic and electromagnetic scattering problems, which has been extended to the inverse scattering problem [30] of determining an inhomogeneous cavity. It should be noted that two reconstruction algorithms $[31,35]$ have also been developed in numerically locating the penetrable medium with embedded acoustic objects inside.

In this paper we shall establish some a priori estimates for both the acoustic and the elastic wave fields by using the integral equation method in Sect. 2. A key role is played by introducing an auxiliary impedance boundary value problem with the boundary data in the sense of $L^{p}$-norm $(1<p<2)$. Section 3 is devoted to the inverse problem of recovering the multi-layered fluid-solid medium. We first determine the surrounding acoustic medium, disregarding the unknown wave number in the medium and the embedded penetrable elastic obstacle. Then we continue to identify the corresponding wave number in the surrounding medium without knowing the embedded penetrable elastic obstacle. Finally, with a knowledge of the surrounding acoustic medium, the unique recovery of the embedded penetrable elastic obstacle will be given.

\section{A priori estimates}

In this section we shall derive some a priori estimates for both the acoustic wave field in the fluid medium and the elastic field in the embedded solid obstacle.

We begin with the formulations of the model problem. Let $D \subset \mathbb{R}^{3}$ be a bounded connected domain, which denotes a penetrable fluid medium with a smooth boundary $\Gamma_{1}:=\partial D \in C^{2}$. Let a bounded and simply connected domain $D_{0} \subset D$ denote an embedded solid obstacle with a smooth boundary $\Gamma_{0}:=\partial D_{0} \in C^{2}$. We further assume that the constant wave number $k_{*}=k_{1} \in \mathbb{R}_{+}$in $D_{1}:=D \backslash \overline{D_{0}}$ and $k_{*}=k \in \mathbb{R}_{+}$in $\mathbb{R}^{3} \backslash \bar{D}$. Moreover, let us denote by $\rho_{f}$ the constant mass density in the fluid medium $D_{1}$ and by $\rho_{s} \in L^{\infty}\left(D_{0}\right)$ the real-valued mass density in the solid obstacle $D_{0}$. See Fig. 1 for the geometric configuration of the scattering problem. 
Figure 1 Geometric configuration of the scattering problem

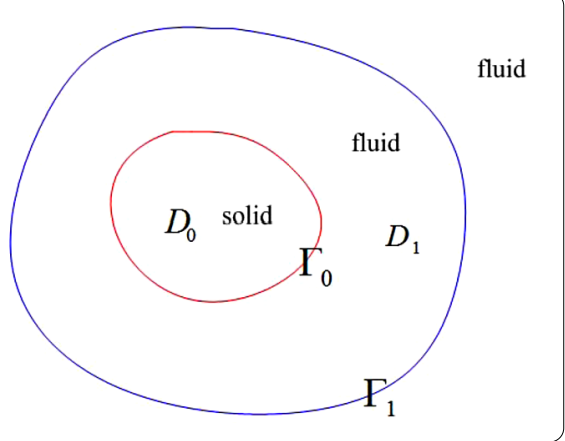

Consider an incident plane wave $p^{i}:=e^{i k x \cdot d}$, where the wave number $k=\omega / c$ with $\omega$ the frequency and $c$ the sound speed, and $d \in \mathbb{S}^{2}:=\left\{x \in \mathbb{R}^{3}:|x|=1\right\}$ is the incident direction. Then the scattering problem can be formulated in determining the total acoustic field $p=p^{i}+p^{s}$ and the transmitted elastic field $\mathbf{u}$ such that

$$
\begin{cases}\triangle p+k_{*}^{2} p=0 & \text { in } \mathbb{R}^{3} \backslash \overline{D_{0}}, \\ \triangle^{*} \mathbf{u}+\rho_{s} \omega^{2} \mathbf{u}=0 & \text { in } D_{0}, \\ t(\mathbf{u})=-p v & \text { on } \Gamma_{0}, \\ \eta \mathbf{u} \cdot v=\frac{\partial p}{\partial v} & \text { on } \Gamma_{0}, \\ \lim _{r \rightarrow \infty} r\left(\frac{\partial p^{s}}{\partial r}-i k p^{s}\right)=0 & \text { with } r=|x|,\end{cases}
$$

where $p^{s}=p-p^{i}$ is the acoustic scattered field, $\Delta^{*}:=\mu \Delta+(\lambda+\mu) \operatorname{grad}$ div with the lamé constants $\lambda, \mu \in \mathbb{R}$ satisfying that $\mu>0,3 \lambda+2 \mu>0, \eta=\rho_{f} \omega^{2}$ and $v$ is the exterior unit normal vector on $\Gamma_{0}$ directed into $D_{1}$. Here the stress vector $t(\mathbf{u})$ on $\Gamma_{0}$ is defined by

$$
t(\mathbf{u}):=2 \mu \frac{\partial \mathbf{u}}{\partial v}+\lambda(\operatorname{div} \mathbf{u}) v+\mu \nu \times \operatorname{curl} \mathbf{u}, \quad \text { on } \Gamma_{0} .
$$

The Sommerfeld radiation condition related to the acoustic scattered field $p^{s}$ in (2.1) enables us to have the following asymptotic behavior:

$$
p^{s}(x)=\frac{e^{i k r}}{4 \pi r}\left\{p_{\infty}(\hat{x})+O\left(\frac{1}{r}\right)\right\} \quad \text { as } r=|x| \rightarrow \infty,
$$

uniformly for all directions $\hat{x}:=x / r \in \mathbb{S}^{2}$, where $p_{\infty}$ is known as the far-field pattern of the acoustic scattered field $p^{s}$.

In order to obtain the a priori estimates for the acoustic field $p$ and the elastic field $\mathbf{u}$ corresponding to different kinds of incident fields, we consider the following scattering problem:

$$
\begin{cases}\triangle q^{s}+k_{*}^{2} q^{s}=0 & \text { in } \mathbb{R}^{3} \backslash \overline{D_{0}}, \\ \frac{\partial q^{s}}{\partial v}+i \beta q^{s}=0 & \text { on } \Gamma_{0}, \\ \lim _{r \rightarrow \infty} r\left(\frac{\partial q^{s}}{\partial r}-i k q^{s}\right)=0 & \text { with } r=|x|,\end{cases}
$$

where $\beta>0$ is a positive constant and the scattered field $q^{s}=q-q^{i}$ with the incident super singular point source $q^{i}:=\nabla_{x} \Phi(x, z) \cdot \vec{a}$ with $z \in \mathbb{R}^{3} \backslash \bar{D}$ and a fixed vector $\vec{a} \in \mathbb{R}^{3}$. Here $\Phi(x, z)=e^{i k|x-z|} / 4 \pi|x-z|$ is the fundamental solution of Helmholtz equation $\Delta w+k^{2} w=0$ in $\mathbb{R}^{3} \backslash\{z\}$. 
We next study the solvability of the scattering problem (2.3) by reformulating it into an equivalent Lippmann-Schwinger-type equation. To accomplish this, we introduce the exterior boundary value problem as follows:

$$
\begin{cases}\Delta v+k^{2} v=0 & \text { in } \mathbb{R}^{3} \backslash \overline{D_{0}}, \\ \frac{\partial v}{\partial v}+i \beta v=h & \text { on } \Gamma_{0}, \\ \lim _{r \rightarrow \infty} r\left(\frac{\partial v}{\partial r}-i k v\right)=0 & \text { with } r=|x| .\end{cases}
$$

Let $G^{s}(\cdot, y)$ be the solution to the problem $(2.4)$ with the boundary data $h=-\partial_{x} \Phi(x, y) / \partial v-$ $i \beta \Phi(x, y)$ with $y \in \mathbb{R}^{3} \backslash \overline{D_{0}}$. Then $G(x, y)=\Phi(x, y)+G^{s}(x, y)$ in $\mathbb{R}^{3} \backslash \overline{D_{0}}$ corresponds to the Green function to the problem (2.4). Furthermore, let $F(\cdot, y)$ be the solution to the problem (2.4) with the boundary data $h=-\partial_{x}\left(\nabla_{x} \Phi(x, y) \cdot \vec{a}\right) / \partial v-i \beta \nabla_{x} \Phi(\cdot, y) \cdot \vec{a}$ with $y \in \mathbb{R}^{3} \backslash \overline{D_{0}}$.

Define $F^{i}:=\nabla_{x} \Phi(x, z) \cdot \vec{a}+F(x, z)$ in $\mathbb{R}^{3} \backslash \overline{D_{0}}$. Then, by the Green representation theorem for the solution $q$ to the problem (2.3) and the radiation conditions for $q^{s}$ and $G^{s}$, it can be shown that the solution $q$ of (2.3) can be represented in the following equivalent Lippmann-Schwinger-type form:

$$
q(x ; z)=F^{i}(x ; z)-\int_{D_{1}}\left(k^{2}-k_{1}^{2}\right) G(x, y) q(y ; z) d y \quad \text { for } x \in D_{1} .
$$

Now define the operator $T$ by

$$
(T \varphi)(x):=\int_{D_{1}}\left(k^{2}-k_{1}^{2}\right) G(x, y) \varphi(y) d y \quad \text { in } D_{1} .
$$

It then follows from (2.5) that

$$
(I+T) q(x ; z)=F^{i}(x ; z) \quad \text { in } L^{s}\left(D_{1}\right) .
$$

Noticing that $F^{i}(\cdot ; z) \in L^{s}\left(D_{1}\right)$ with $6 / 5 \leq s<3 / 2$. It is known from [11, Theorem 9.9] that $T$ is bounded from $L^{s}\left(D_{1}\right)$ into $W^{2, s}\left(D_{1}\right)$, so it is compact from $L^{s}\left(D_{1}\right)$ to itself. Hence, the operator $I+T$ is of Fredholm type with index zero due to the uniqueness of the problem (2.3). Then an application of the Fredholm alternative implies that there exists a unique solution $q$ to the problem (2.3) satisfying that

$$
\|q(\cdot ; z)\|_{L^{s}\left(D_{1}\right)} \leq C\left\|F^{i}(\cdot ; z)\right\|_{L^{s}\left(D_{1}\right)} \leq C\left\|q^{i}(\cdot ; z)\right\|_{L^{s}\left(D_{1}\right)}
$$

with $6 / 5 \leq s<3 / 2$.

In addition, it is noted that

$$
q(x ; z)-F^{i}(x ; z)=-T q(x ; z) \quad \text { for } x \in D_{1} .
$$

This, together with the fact that $T q \in W^{2, s}\left(D_{1}\right) \hookrightarrow H^{1}\left(D_{1}\right)$ for $6 / 5 \leq s<3 / 2$, gives

$$
\left\|q-F^{i}\right\|_{H^{1}\left(D_{1}\right)} \leq C\left\|q^{i}\right\|_{L^{s}\left(D_{1}\right)} \quad \text { with } 6 / 5 \leq s<3 / 2
$$

Combining (2.7) and (2.9) yields the following uniform a priori estimate for the solution $q$ of the problem (2.3). 
Theorem 2.1 For $z^{*} \in \Gamma_{1}$, let $B_{\varepsilon_{0}}\left(z^{*}\right)$ be a small ball centered at $z^{*}$ with the radius $\varepsilon_{0}>0$. Let $z \in B_{\varepsilon_{0}}\left(z^{*}\right) \cap\left(\mathbb{R}^{3} \backslash \bar{D}\right)$ and let $q$ be the solution to the problem (2.3) produced by the incident supper singular point source $q^{i}=\nabla_{x} \Phi(x, z) \cdot \vec{a}$, where $\vec{a} \in \mathbb{R}^{3}$ is a fixed vector. Then $q \in L^{s}\left(D_{1}\right)$ and $q-q^{i} \in H^{1}\left(D_{1}\right)$ with the estimate

$$
\|q\|_{L^{s}\left(D_{1}\right)}+\left\|q-q^{i}\right\|_{H^{1}\left(D_{1}\right)} \leq C\left\|q^{i}\right\|_{L^{s}\left(D_{1}\right)}
$$

where $6 / 5 \leq s<3 / 2$ and $C>0$ is a positive constant independent of $z$.

Remark 2.2 For a general incident field $q^{i}$ such as a plane wave $q^{i}=e^{i k x \cdot d}$ with $d \in \mathbb{S}^{2}$ or a point source $q^{i}=\Phi(x, z)$ with $z \in \mathbb{R}^{3} \backslash \bar{D}$ or a point source $q^{i}=\Phi_{1}(x, z)=e^{i k_{1}|x-z|} / 4 \pi|x-z|$ with $z \in D_{1}$, it can be deduced from Theorem 2.1 that the solution $q$ of (2.3) satisfies

$$
\|q\|_{L^{2}\left(D_{1}\right)}+\left\|q-q^{i}\right\|_{H^{1}\left(D_{1}\right)} \leq C\left\|q^{i}\right\|_{L^{2}\left(D_{1}\right)}
$$

for a positive constant $C>0$.

Before going further, we here remark that $\omega \in \mathbb{R}$ is called a Jones frequency if the following boundary value problem:

$$
\begin{cases}\triangle^{*} \mathbf{u}+\rho_{s} \omega^{2} \mathbf{u}=0 & \text { in } D_{0}, \\ t(\mathbf{u})=0 & \text { on } \Gamma_{0}, \\ \eta \mathbf{u} \cdot v=0 & \text { on } \Gamma_{0}\end{cases}
$$

has a nontrivial solution, that is, $\mathbf{u} \neq 0$ in $D_{0}$ (cf. $[17,24]$ ).

Now, we consider the general boundary value problem

$$
\begin{cases}\Delta \widetilde{w}+k_{*}^{2} \widetilde{w}=0 & \text { in } \mathbb{R}^{3} \backslash \overline{D_{0}}, \\ \triangle^{*} \widetilde{\mathbf{u}}+\rho_{s} \omega^{2} \widetilde{\mathbf{u}}=0 & \text { in } D_{0}, \\ t(\widetilde{\mathbf{u}})+\widetilde{w} v=\mathbf{f}_{1} & \text { on } \Gamma_{0}, \\ \eta \widetilde{\mathbf{u}} \cdot v-\frac{\partial \widetilde{w}}{\partial v}=f_{2} & \text { on } \Gamma_{0}, \\ \lim _{r \rightarrow \infty} r\left(\frac{\partial \widetilde{w}}{\partial r}-i k \widetilde{w}\right)=0 & \text { with } r=|x|,\end{cases}
$$

where $\mathbf{f}_{1} \in H^{-1 / 2}\left(\Gamma_{0}\right)^{3}, f_{2} \in H^{-1 / 2}\left(\Gamma_{0}\right)$. If $\omega \in \mathbb{R}$ is not a Jones frequency, using the coupling method of a variational and boundary integral equation techniques (cf. [13, 20]), the wellposedness of (2.13) can be obtained, which is stated as follows.

Theorem 2.3 If $\omega \in \mathbb{R}$ is not a Jones frequency, then the problem (2.13) admits a unique solution $(\widetilde{w}, \widetilde{\mathbf{u}}) \in H_{\text {loc }}^{1}\left(\mathbb{R}^{3} \backslash \overline{D_{0}}\right) \times H^{1}\left(D_{0}\right)^{3}$ satisfying

$$
\|\widetilde{w}\|_{H^{1}\left(B_{R}\right\rangle \overline{\left.D_{0}\right)}}+\|\widetilde{\mathbf{u}}\|_{H^{1}\left(D_{0}\right)^{3}} \leq C\left(\left\|\mathbf{f}_{1}\right\|_{H^{-1 / 2}\left(\Gamma_{0}\right)^{3}}+\left\|f_{2}\right\|_{H^{-1 / 2}\left(\Gamma_{0}\right)}\right)
$$

for any sufficiently large $R>0$. Here $B_{R}:=\left\{x \in \mathbb{R}^{3}:|x| \leq R\right\}$ and $C>0$ is a constant depending on $R$.

Based on Theorem 2.1 and 2.3, we next establish the a priori estimate for the problem (2.1). Let $(p, \mathbf{u})$ be the solution to the problem (2.1) corresponding to the incident super 
singular point source $p^{i}=\nabla_{x} \Phi(x, z) \cdot \vec{a}$ with $z \in \mathbb{R}^{3} \backslash \bar{D}$ and a fixed vector $\vec{a} \in \mathbb{R}^{3}$. Assume that $q$ is the total field corresponding to the scattered field $q^{s}$ in (2.1) with the incident super singular point source $q^{i}:=\nabla_{x} \Phi(x, z) \cdot \vec{a}$. Let $\widetilde{w}:=p-q$ in $\mathbb{R}^{3} \backslash \overline{D_{0}}$ and $\widetilde{\mathbf{u}}=\mathbf{u}$ in $D_{0}$, then $(\widetilde{w}, \widetilde{\mathbf{u}})$ is the solution to the problem (2.13) with the boundary data $\mathbf{f}_{1}:=-q v=$ $-\left[\left(q-q^{i}\right)+q^{i}\right] v$ and $f_{2}:=-i \beta q=-i \beta\left[\left(q-q^{i}\right)+q^{i}\right]$. Moreover, it is noted from Theorem 2.1 that $q-q^{i} \in H^{1}\left(D_{1}\right)$ and $q^{i}=\nabla_{x} \Phi(x, z) \cdot \vec{a}$ with $z \in \mathbb{R}^{3} \backslash \bar{D}$. By the trace theorem and the positive distance between $z$ and $\Gamma_{0}$, it is deduced that $\mathbf{f}_{1} \in H^{-1 / 2}\left(\Gamma_{0}\right)^{3}$ and $f_{2} \in H^{-1 / 2}\left(\Gamma_{0}\right)$, respectively. Therefore, by Theorem 2.1 and 2.3, we have the following theorem.

Theorem 2.4 For $z^{*} \in \Gamma_{1}$, let $B_{\varepsilon_{0}}\left(z^{*}\right)$ be a small ball centered at $z^{*}$ with the radius $\varepsilon_{0}>0$. Let $z \in B_{\varepsilon_{0}}\left(z^{*}\right) \cap\left(\mathbb{R}^{3} \backslash \bar{D}\right)$ and let $(p, \mathbf{u})$ be the solution to the problem (2.1) corresponding to the incident super singular point source $p^{i}=\nabla_{x} \Phi(x, z) \cdot \vec{a}$ with a fixed vector $\vec{a} \in \mathbb{R}^{3}$. Then $p \in L^{s}\left(D_{1}\right)$ and $p-p^{i} \in H^{1}\left(D_{1}\right)$ satisfy

$$
\|p\|_{L^{s}\left(D_{1}\right)}+\left\|p-p^{i}\right\|_{H^{1}\left(D_{1}\right)} \leq C\left\|p^{i}\right\|_{L^{s}\left(D_{1}\right)}
$$

where $6 / 5 \leq s<3 / 2$ and $C>0$ is a positive constant independent of $z$.

Remark 2.5 For a general incident field $p^{i}$ such as a plane wave $p^{i}=e^{i k x \cdot d}$ with $d \in \mathbb{S}^{2}$ or a point source $p^{i}=\Phi(x, z)$ with $z \in \mathbb{R}^{3} \backslash \bar{D}$, it can be verified from Theorems 2.1-2.4 that the acoustic wave field $p$ of the problem (2.1) satisfies

$$
\|p\|_{L^{2}\left(D_{1}\right)}+\left\|p-p^{i}\right\|_{H^{1}\left(D_{1}\right)} \leq C\left\|p^{i}\right\|_{L^{2}\left(D_{1}\right)}
$$

for a positive constant $C>0$.

Relying on the above arguments, we can also derive the a priori estimate for the elastic wave field $\mathbf{u}$ of the problem (2.1) in the next theorem.

Theorem 2.6 For $z^{*} \in \Gamma_{0}$, let $B_{\varepsilon_{0}}\left(z^{*}\right)$ be a small ball centered at $z^{*}$ with the radius $\varepsilon_{0}>0$. Let $z \in B_{\varepsilon_{0}}\left(z^{*}\right) \cap\left(\mathbb{R}^{3} \backslash \overline{D_{0}}\right)$ and let $(p, \mathbf{u})$ be the solution to the problem (2.1) corresponding to the incident point source $p^{i}=\Phi_{1}(x, z)$. Then we have

$$
\|\mathbf{u}\|_{H^{1}\left(D_{0}\right)^{3}} \leq C,
$$

where $C>0$ is a positive constant independent of $z$.

Proof Let $q$ be the solution to the problem (2.3) generated by the incident point source $q^{i}=\Phi_{1}(\cdot, z)$ with $z \in B_{\varepsilon_{0}}\left(z^{*}\right) \cap\left(\mathbb{R}^{3} \backslash \overline{D_{0}}\right)$. Let $\widetilde{w}:=p-q$ in $\mathbb{R}^{3} \backslash \overline{D_{0}}$ and $\widetilde{\mathbf{u}}=\mathbf{u}$ in $D_{0}$, then $(\widetilde{w}, \widetilde{\mathbf{u}})$ is the solution to the problem (2.13) with the boundary data $\mathbf{f}_{1}:=-q v=-\left(q-q^{i}\right) v-$ $q^{i} v$ and $f_{2}:=-i \beta\left(q-q^{i}\right)-i \beta q^{i}$. It is known from Remark 2.2 that $q-q^{i} \in H^{1}\left(D_{1}\right)$. This together with the fact that $q^{i}=\Phi_{1}(\cdot, z) \in L^{s}\left(\Gamma_{0}\right)$ with $4 / 3<s<2$ further implies that $\mathbf{f}_{1} \in$ $H^{-1 / 2}\left(\Gamma_{0}\right)^{3}$ and $f_{2} \in H^{-1 / 2}\left(\Gamma_{0}\right)$, respectively. Therefore, the required estimate (2.16) follows from Theorem 2.3. This completes the proof of the theorem. 


\section{Recovery of $\Gamma_{1}, k_{1}$ and $\Gamma_{0}$}

In this section we focus on the global uniqueness issue of the inverse problem consisting of the identification of the interface $\Gamma_{1}$ of the fluid medium, the corresponding wave number $k_{1}$ and the interface $\Gamma_{0}$ between fluid and solid bodies. The proof is mainly based on constructing different kinds of well-posed interior transmission problems associated with the Helmholtz equation and the modified interior transmission problem related to the coupled system of the Helmholtz equation and the Navier equation, and the a priori estimates established in Sect. 2.

We state the global uniqueness result in this paper on our inverse problem.

Theorem 3.1 Let $\left(D, \Gamma_{0}, k_{1}, \Gamma_{1}\right)$ and $\left(\widetilde{D}, \widetilde{\Gamma}_{0}, \widetilde{k}_{1}, \widetilde{\Gamma}_{1}\right)$ be two scatterers. Suppose that $p_{\infty}(\hat{x} ; d)$ and $\widetilde{p}_{\infty}(\hat{x} ; d)$ are the far-field patterns of the acoustic scattering solution to the scatterers $\left(D, \Gamma_{0}, k_{1}, \Gamma_{1}\right)$ and $\left(\widetilde{D}, \widetilde{\Gamma}_{0}, \widetilde{k}_{1}, \widetilde{\Gamma}_{1}\right)$, respectively, corresponding to the incident plane wave $p^{i}(x)=e^{i k x \cdot d}, d \in \mathbb{S}^{2}$. Suppose further that $\omega$ is not a Jones frequency for $\left(D, \Gamma_{0}, k_{1}, \Gamma_{1}\right)$ and $\left(\widetilde{D}, \widetilde{\Gamma}_{0}, \widetilde{k}_{1}, \widetilde{\Gamma}_{1}\right)$. Assume that $p_{\infty}(\hat{x} ; d)=\widetilde{p}_{\infty}(\hat{x} ; d)$ for all $\hat{x}, d \in \mathbb{S}^{2}$, then $\Gamma_{1}=\widetilde{\Gamma}_{1}, k_{1}=\widetilde{k}_{1}$ and $\Gamma_{0}=\widetilde{\Gamma}_{0}$.

Proof Step 1. We first prove $\Gamma_{1}=\widetilde{\Gamma}_{1}$ by contradiction. Assume on the contrary that $\Gamma_{1} \neq$ $\widetilde{\Gamma}_{1}$. Let $G$ denote the unbounded connected part of $\mathbb{R}^{3} \backslash \overline{(D \cup \widetilde{D})}$. Without loss of generality, there exists at least a point $z^{*} \in \Gamma_{1} \cap \partial G$ and $z^{*} \notin \widetilde{\Gamma}_{1}$. We now define

$$
z_{j}:=z^{*}+\frac{\delta}{j} v\left(z^{*}\right), \quad j=1,2,3, \ldots,
$$

with sufficiently small $\delta>0$ such that $z_{j} \in B_{\varepsilon_{0}}\left(z^{*}\right) \cap G$ for all $j \in \mathbb{N}$. Here $B_{\varepsilon_{0}}\left(z^{*}\right)$ denotes a small ball centered at $z^{*}$ with the radii $\varepsilon_{0}>0$ satisfying that $B_{\varepsilon_{0}}\left(z^{*}\right) \cap \widetilde{D}=\emptyset$ and $B_{\varepsilon_{0}}\left(z^{*}\right) \cap$ $D_{0}=\emptyset$.

Let $\left(p_{j}, \mathbf{u}_{j}\right)$ and $\left(\widetilde{p}_{j}, \widetilde{\mathbf{u}}_{j}\right)$ be the solutions to the problem (2.1) with respect to the scatterers $\left(D, \Gamma_{0}, k_{1}, \Gamma_{1}\right)$ and $\left(\widetilde{D}, \widetilde{\Gamma}_{0}, \widetilde{k}_{1}, \widetilde{\Gamma}_{1}\right)$, respectively, generated by the same incident super singular point source

$$
p_{j}^{i}:=\nabla_{x} \Phi\left(x, z_{j}\right) \cdot v\left(z^{*}\right), \quad j=1,2,3, \ldots
$$

By applying Rellich's lemma and the denseness analysis [4, Theorems 5.4, 5.5] or [24, Theorem 4.1$]$, we drive from the assumption that $p_{\infty}(\hat{x} ; d)=\widetilde{p}_{\infty}(\hat{x} ; d)$ for all $\hat{x}, d \in \mathbb{S}^{2}$ that

$$
p_{j}^{s}(x)=\widetilde{p}_{j}^{s}(x) \quad \text { in } \bar{G}, j=1,2,3, \ldots,
$$

where $p_{j}^{s}=p_{j}-p_{j}^{i}$ and $\widetilde{p}_{j}^{s}=\widetilde{p}_{j}-p_{j}^{i}$ are the corresponding scattered fields.

Since $z^{*} \in \Gamma_{1}$ and $\Gamma_{1}$ is of $C^{2}$-class, we can choose a small $C^{2}$-smooth domain $\Omega_{*}$ satisfying that $\left(B_{\varepsilon_{0}}\left(z^{*}\right) \cap D_{1}\right) \subset \Omega_{*} \subset\left(D_{1} \backslash \widetilde{D}\right)$. Moreover, it is noted that the smallest Dirichlet eigenvalue $\lambda_{1}\left(\Omega_{*}\right)$ of $-\triangle$ tends to $+\infty$ as the diameter $\rho \rightarrow 0$. Then the diameter $\rho$ of $\Omega_{*}$ can also be chosen sufficiently small such that $k^{2}<\min \left\{\lambda_{1}\left(D_{0}\right), k^{2} \lambda_{1}\left(D_{0}\right) / k_{1}^{2}\right\}$, which implies that $k^{2}$ is not an eigenvalue of the following interior transmission problem:

$$
\begin{cases}\triangle w_{1}+k^{2} w_{1}=0 & \text { in } \Omega_{*}, \\ \Delta w_{2}+k_{1}^{2} w_{2}=0 & \text { in } \Omega_{*}, \\ w_{1}-w_{2}=f_{1} & \text { on } \partial \Omega_{*}, \\ \frac{\partial w_{1}}{\partial v}-\frac{\partial w_{2}}{\partial v}=f_{2} & \text { on } \partial \Omega_{*},\end{cases}
$$


in the case when $f_{1}=0$ and $f_{2}=0$. Moreover, it is known from [3] that the problem (3.3) admits a unique solution $\left(w_{1}, w_{2}\right) \in L^{2}\left(\Omega_{*}\right) \times L^{2}\left(\Omega_{*}\right)$ with the estimate

$$
\left\|w_{1}\right\|_{\left.L^{2} \Omega_{*}\right)}+\left\|w_{2}\right\|_{L^{2}\left(\Omega_{*}\right)} \leq C\left\|\left(f_{2}, f_{1}\right)\right\|_{-\frac{1}{2} \times \frac{1}{2}}
$$

assuming $f_{1}$ and $f_{2}$ satisfy the condition: For $f_{1} \in H^{\frac{1}{2}}\left(\partial \Omega_{*}\right)$ and $f_{2} \in H^{-\frac{1}{2}}\left(\partial \Omega_{*}\right)$, there exists a function $h_{f} \in H_{\Delta}^{1}\left(\Omega_{*}\right):=\left\{h \in H^{1}\left(\Omega_{*}\right): \Delta h \in L^{2}\left(\Omega_{*}\right)\right\}$ satisfying $h_{f}=f_{1}$ and $\partial h_{f} / \partial v=f_{2}$ on $\partial \Omega_{*}$. Here

$$
\left\|\left(f_{2}, f_{1}\right)\right\|_{-\frac{1}{2} \times \frac{1}{2}}:=\inf _{h_{f} \in H_{\triangle}^{1}\left(\Omega_{*}\right) h_{f}=f_{1} \partial h_{f} / \partial v=f_{2}}\left\{\left\|h_{f}\right\|_{H^{1}\left(\Omega_{*}\right)}+\left\|\Delta h_{f}\right\|_{L^{2}\left(\Omega_{*}\right)}\right\} .
$$

Furthermore, let $\left(w_{1, j}, w_{2, j}\right):=\left(\widetilde{p}_{j}, p_{j}\right)$ in $\Omega_{*}$. Then it is easily verified that $\left(w_{1 j}, w_{2 j}\right)$ is the unique solution of the problem (3.3) with the boundary data

$$
\begin{aligned}
& f_{1}:=f_{1, j}=\widetilde{p}_{j}-p_{j} \quad \text { on } \partial \Omega_{*}, \\
& f_{2}:=f_{2, j}=\frac{\partial \widetilde{p}_{j}}{\partial v}-\frac{\partial p_{j}}{\partial v} \quad \text { on } \partial \Omega_{*} .
\end{aligned}
$$

Clearly, we have from (3.2)

$$
f_{1, j}=0 \quad \text { and } \quad f_{2, j}=0, \quad \text { on } \partial \Omega_{*} \cap B_{\varepsilon_{0}}\left(z_{*}\right) .
$$

Next, we shall prove that $\left(f_{2, j}, f_{1, j}\right)$ satisfies the condition. To accomplish this, we define a cut-off function $\chi(x) \in C^{2}\left(\mathbb{R}^{3}\right)$ supported in $B_{\varepsilon_{1}}\left(z^{*}\right)$ with $\varepsilon_{1}<\varepsilon_{0}$ and satisfying that $\chi(x)=1$ in $B_{\varepsilon_{2}}\left(z^{*}\right)$ with some fixed $\varepsilon_{2}<\varepsilon_{1}$. Then we define a function $h_{j}(x)$ by

$$
h_{j}(x):=(1-\chi(x))\left(\widetilde{p}_{j}(x)-p_{j}(x)\right) \quad \text { in } \Omega_{*} .
$$

Obviously, it is found from (3.6) and (3.7) that $h_{j}=f_{1, j}$ and $\partial h_{j} / \partial v=f_{2, j}$ on $\partial \Omega_{*}$. Furthermore, we obtain $h_{j} \in H_{\Delta}^{1}\left(\Omega_{*}\right)$ for any fixed $j \in \mathbb{N}$ due to the fact that $z_{j} \in B_{\varepsilon_{0}}\left(z^{*}\right) \cap \partial G$. Therefore, $\left(f_{2, j}, f_{1, j}\right)$ satisfies the condition between (3.4) and (3.5) for any fixed $j \in \mathbb{N}$.

In the following, it remains to show the uniform boundedness of $\left(f_{2, j}, f_{1, j}\right)$ in the norm given by (3.5) for all $j \in \mathbb{N}$. It is first noted that

$$
\left\|\tilde{p}_{j}^{s}\right\|_{H^{1}\left(\Omega_{*}\right)}+\left\|\Delta \widetilde{p}_{j}^{s}\right\|_{L^{2}\left(\Omega_{*}\right)} \leq C_{1}
$$

since $z^{*}$ has a positive distance from $\widetilde{\Gamma}_{1}$ and the acoustic wave field $\widetilde{p}_{j}$ satisfies the Helmholtz equation $\triangle \widetilde{p}_{j}+k^{2} \widetilde{p}_{j}=0$ in $\Omega_{*}$. It is then deduced from the definition of $h_{j}$ that

$$
\begin{aligned}
& \left\|h_{j}\right\|_{H^{1}\left(\Omega_{*}\right)}+\left\|\Delta h_{j}\right\|_{L^{2}\left(\Omega_{*}\right)} \\
& \quad \leq C\left(\left\|p_{j}^{s}\right\|_{H^{1}\left(\Omega_{*} \backslash \overline{\left.B_{\varepsilon_{2}}\left(z^{*}\right)\right)}\right.}+\left\|\Delta p_{j}^{s}\right\|_{L^{2}\left(\Omega_{*} \backslash \overline{\left.B_{\varepsilon_{2}}\left(z^{*}\right)\right)}\right.}+C_{1}\right) \\
& \quad \leq C\left(k_{1}^{2}\right)\left\|p_{j}\right\|_{H^{1}\left(\Omega_{*} \backslash \overline{B_{\varepsilon_{2}}\left(z^{*}\right)}\right)}
\end{aligned}
$$

uniformly for all $j \in \mathbb{N}$. Hence, by Theorem 2.4 we deduce that

$$
\left\|\left(f_{2, j}, f_{1, j}\right)\right\|_{-\frac{1}{2} \times \frac{1}{2}} \leq C\left\|h_{j}\right\|_{H^{1}\left(\Omega_{*}\right)}+\left\|\Delta h_{j}\right\|_{L^{2}\left(\Omega_{*}\right)} \leq C
$$


uniformly for all $j \in \mathbb{N}$. This, together with (3.4) gives

$$
\left\|w_{1, j}\right\|_{\left.L^{2} \Omega_{*}\right)}+\left\|w_{2, j}\right\|_{L^{2}\left(\Omega_{*}\right)} \leq C \quad \text { uniformly for all } j \in \mathbb{N} \text {. }
$$

Consequently, we have

$$
\left\|p_{j}^{i}\right\|_{L^{2}\left(\Omega_{*}\right)}-\left\|p_{j}-p_{j}^{i}\right\|_{L^{2}\left(\Omega_{*}\right)} \leq\left\|w_{2, j}\right\|_{L^{2}\left(\Omega_{*}\right)} \leq C
$$

Moreover, it can be easily checked that

$$
\left\|p_{j}^{i}\right\|_{L^{2}\left(\Omega_{*}\right)}^{2}=\int_{\Omega_{*}}\left|\nabla_{x} \Phi\left(\cdot, z_{j}\right) \cdot v\left(z^{*}\right)\right|^{2} d x \geq C \frac{1}{j^{2}} \int_{\Omega_{*}} \frac{1}{\left|x-z_{j}\right|^{6}} d x=O(j)
$$

Then we see that (3.13) is a contradiction since $\left\|p_{j}^{i}\right\|_{L^{2}\left(\Omega_{*}\right)} \rightarrow \infty$ as $j \rightarrow \infty$ and $\| p_{j}-$ $p_{j}^{i} \|_{L^{2}\left(\Omega_{*}\right)}$ is uniformly bounded due to Theorem 2.4. Therefore, we get $\Gamma_{1}=\widetilde{\Gamma}_{1}$.

Step 2 . We continue to prove $k_{1}=\widetilde{k}_{1}$ by contradiction. Suppose that $k_{1} \neq \widetilde{k}_{1}$. Then we can choose $x^{*} \in \Gamma_{1}$ and define

$$
x_{j}:=x^{*}+\frac{\delta}{j} v\left(x^{*}\right), \quad j=1,2,3, \ldots
$$

with sufficiently small $\delta>0$ such that $x_{j} \in B_{\varepsilon}\left(x^{*}\right) \cap\left(\mathbb{R}^{3} \backslash \bar{D}\right)$, where $B_{\varepsilon}\left(x^{*}\right)$ denotes a ball centered at $x^{*}$ with the radii $\varepsilon>0$ satisfying that $B_{\varepsilon}\left(x^{*}\right) \cap D_{0}=\emptyset$ and $B_{\varepsilon}\left(x^{*}\right) \cap \widetilde{D}_{0}=\emptyset$. We further define $\Omega_{1}:=B_{\varepsilon}\left(x^{*}\right) \cap\left(D_{1} \cap \widetilde{D}_{1}\right)$.

Now, we still let $\left(p_{j}, \mathbf{u}_{j}\right)$ and $\left(\widetilde{p}_{j}, \widetilde{\mathbf{u}}_{j}\right)$ be the solutions to the problem (2.1) with respect to the scatterers $\left(D, \Gamma_{0}, k_{1}, \Gamma_{1}\right)$ and $\left(\widetilde{D}, \widetilde{\Gamma}_{0}, \widetilde{k}_{1}, \widetilde{\Gamma}_{1}\right)$, respectively, generated by the incident super singular point source $p_{j}^{i}$ defined by (3.1) with $z_{j}$ replaced by $x_{j}$. It then follows that $\left(v_{1, j}, v_{2, j}\right):=\left(\widetilde{p}_{j}, p_{j}\right)$ solves the following interior transmission problem:

$$
\begin{cases}\Delta v_{1, j}+\widetilde{k}_{1}^{2} v_{1, j}=0 & \text { in } \Omega_{1}, \\ \Delta v_{2, j}+k_{1}^{2} v_{2, j}=0 & \text { in } \Omega_{1}, \\ v_{1, j}-v_{2, j}=h_{1, j} & \text { on } \partial \Omega_{1}, \\ \frac{\partial v_{1, j}}{\partial v}-\frac{\partial v_{2, j}}{\partial v}=h_{2, j} & \text { on } \partial \Omega_{1},\end{cases}
$$

where $h_{1, j}, h_{2, j}$ are defined as $f_{1, j}, f_{2, j}$ in (3.6) and (3.7). Arguing similarly as those between (3.8) and (3.14), we arrive at a contradiction. Then we have proved $k_{1}=\widetilde{k}_{1}$.

Step 3. We finally show the interface $\Gamma_{0}=\widetilde{\Gamma}_{0}$ by contradiction. Assume on the contrary that $\Gamma_{0} \neq \widetilde{\Gamma}_{0}$. Without loss of generality, we can choose $y^{*} \in \Gamma_{0}$ but $y^{*} \notin \widetilde{\Gamma}_{0}$. Define

$$
y_{j}:=y^{*}+\frac{\delta}{j} v\left(y^{*}\right), \quad j=1,2,3, \ldots
$$

with sufficiently small $\delta>0$ such that $z_{j} \in B_{\varepsilon}\left(y^{*}\right) \cap G_{0}$ for all $j \in \mathbb{N}$. Here $G_{0}$ is the unbounded connected part of $\mathbb{R}^{3} \backslash \overline{\left(D_{0} \cup \widetilde{D}_{0}\right)}$ and $B_{\varepsilon}\left(y^{*}\right)$ denotes a small ball centered at $y^{*}$ with the radii $\varepsilon>0$ satisfying that $B_{\varepsilon}\left(y^{*}\right) \cap \widetilde{D}_{0}=\emptyset$. Then we can choose a small $C^{2}$-smooth domain $\Omega_{0}$ such that $\left(B_{\varepsilon}\left(y^{*}\right) \cap D_{0}\right) \subset \Omega_{0} \subset\left(D_{0} \backslash{\widetilde{D_{0}}}_{0}\right) \cap \widetilde{D}_{1}$.

We still denote by $\left(p_{j}, \mathbf{u}_{j}\right)$ and $\left(\widetilde{p}_{j}, \widetilde{\mathbf{u}}_{j}\right)$ the solutions to the problem (2.1) with respect to the scatterers $\left(D, \Gamma_{0}, k_{1}, \Gamma_{1}\right)$ and $\left(\widetilde{D}, \widetilde{\Gamma}_{0}, \widetilde{k}_{1}, \widetilde{\Gamma}_{1}\right)$, respectively, corresponding to the general 
incident point source

$$
p_{j}^{i}(x):=\Phi_{1}\left(x, y_{j}\right)=\frac{e^{i k_{1}\left|x-y_{j}\right|}}{4 \pi\left|x-y_{j}\right|}, \quad j=1,2,3, \ldots,
$$

where $\Phi_{1}\left(x, y_{j}\right)$ is the fundamental solution of the Helmholtz equation $\Delta \Phi_{1}+k_{1}^{2} \Phi_{1}=$ $-\delta\left(\cdot-y_{j}\right)$ in $\mathbb{R}^{3}$. Firstly, by using Rellich's lemma and the mixed reciprocity relation in $[4$, Theorem 3.16], we deduce that

$$
p_{j}(x)=\widetilde{p}_{j}(x) \quad \text { in } \overline{G_{0}}, j=1,2,3, \ldots
$$

Moreover, let $v_{j}:=\widetilde{p}_{j}$ in $\Omega_{0}$ and $\mathbf{w}_{j}:=\mathbf{u}_{j}$ in $\Omega_{0}$. It can be verified that $\left(v_{j}, \mathbf{w}_{j}\right)$ satisfies the following modified interior transmission problem:

$$
\begin{cases}\Delta v_{j}-a_{1} v_{j}=g_{1, j} & \text { in } \Omega_{0}, \\ \triangle^{*} \mathbf{w}_{j}-a_{2} \mathbf{w}_{j}=\mathbf{g}_{2, j} & \text { in } \Omega_{0}, \\ t\left(\mathbf{w}_{j}\right)+v_{j} v=\mathbf{f}_{j} & \text { on } \partial \Omega_{0}, \\ \eta \mathbf{w}_{j} \cdot v-\partial v_{j} / \partial v=h_{j} & \text { on } \partial \Omega_{0},\end{cases}
$$

with two positive constants $a_{1}, a_{2}>0$ and the boundary data

$$
\begin{aligned}
& g_{1, j}:=-\left(k_{1}^{2}+a_{1}\right) \widetilde{p}_{j}, \quad \mathbf{g}_{2, j}:=-\left(\rho_{s} \omega^{2}+a_{2}\right) \mathbf{u}_{j}, \\
& \mathbf{f}_{j}:=t\left(\mathbf{u}_{j}\right)+\widetilde{p}_{j} v, \quad h_{j}:=\eta \mathbf{u}_{j} \cdot v-\partial \widetilde{p}_{j} / \partial v .
\end{aligned}
$$

Clearly, we deduce from (3.17) that

$$
\mathbf{f}_{j}=0 \quad \text { and } \quad h_{j}=0, \quad \text { on } \Sigma_{0}:=\partial \Omega_{0} \cap \partial G_{0} .
$$

For the problem (3.18), it was shown in [24] (see [24, Theorem 3.2]) that (3.18) is wellposed and its solution $\left(v_{j}, \mathbf{w}_{j}\right)$ satisfies the a priori estimate

$$
\begin{aligned}
& \left\|v_{j}\right\|_{H^{1}\left(\Omega_{0}\right)}+\left\|\mathbf{w}_{j}\right\|_{H^{1}\left(\Omega_{0}\right)^{3}} \\
& \quad \leq C_{0}\left(\left\|g_{1, j}\right\|_{L^{2}\left(\Omega_{0}\right)}+\left\|\mathbf{g}_{2, j}\right\|_{L^{2}\left(\Omega_{0}\right)^{3}}+\left\|\mathbf{f}_{j}\right\|_{H^{-1 / 2}\left(\partial \Omega_{0}\right)^{3}}+\left\|h_{j}\right\|_{H^{-1 / 2}\left(\partial \Omega_{0}\right)}\right)
\end{aligned}
$$

for a positive constant $C_{0}$ independent of $g_{1, j}, \mathbf{g}_{2, j}, \mathbf{f}_{j}, h_{j}$ and $j \in \mathbb{N}$.

We next prove the uniform boundedness of $g_{1, j}, \mathbf{g}_{2, j}, \mathbf{f}_{j}$ and $h_{j}$ in the corresponding Hilbert space for all $j \in \mathbb{N}$.

It is observed from Theorem 2.6 that $\mathbf{u}_{j}$ is uniformly bounded in $H^{1}\left(\Omega_{0}\right)^{3}$, which gives the uniform boundedness of $\mathbf{g}_{2, j}$ in $L^{2}\left(\Omega_{0}\right)^{3}$ for all $j \in \mathbb{N}$. Thanks to the positive distance between $y^{*}$ and $\widetilde{\Gamma}_{0}$, we obtain

$$
\left\|\widetilde{p}_{j}\right\|_{L^{2}\left(\Omega_{0}\right)}+\left\|\widetilde{p}_{j}^{s}\right\|_{H^{1}\left(\Omega_{0}\right)} \leq C \quad \text { uniformly for all } j \in \mathbb{N} \text {. }
$$

This immediately shows that $g_{1, j}$ is uniformly bounded in $L^{2}\left(\Omega_{0}\right)$ for all $j \in \mathbb{N}$. Furthermore, with the aid of (3.19), we only need to prove the uniform boundedness of $\left\|\mathbf{f}_{j}\right\|_{H^{-1 / 2}\left(\partial \Omega_{0} \backslash \Sigma_{0}\right)^{3}}$ and $\left\|h_{j}\right\|_{H^{-1 / 2}\left(\partial \Omega_{0} \backslash \Sigma_{0}\right)}$ for all $j \in \mathbb{N}$. By the trace theorem, it is then sufficient to show the 
uniform boundedness of $\left\|\mathbf{u}_{j}\right\|_{H^{1}\left(\Omega_{0} \backslash \overline{\left.B_{\varepsilon}\left(y^{*}\right)\right)^{3}}\right.}$ and $\left\|\widetilde{p}_{j}\right\|_{H^{1}\left(\Omega_{0} \backslash \overline{\left.B_{\varepsilon}\left(y^{*}\right)\right)}\right.}$ for all $j \in \mathbb{N}$. In fact, noting that $\Phi_{1}\left(x, y_{j}\right) \in H^{1}\left(\Omega_{0} \backslash \overline{B_{\varepsilon}\left(y^{*}\right)}\right)$ uniformly for all $j \in \mathbb{N}$. This, combined with (3.21) and the fact that $\widetilde{p}_{j}=\Phi_{1}\left(\cdot, y_{j}\right)+\widetilde{p}_{j}^{s}$, implies $\widetilde{p}_{j} \in H^{1}\left(\Omega_{0} \backslash \overline{B_{\varepsilon}\left(y^{*}\right)}\right)$ uniformly for all $j \in \mathbb{N}$. The uniform boundedness of $\left\|\mathbf{u}_{j}\right\|_{H^{1}\left(\Omega_{0} \backslash \overline{\left.B_{\varepsilon}\left(y^{*}\right)\right)^{3}}\right.}$ for all $j \in \mathbb{N}$ follows from Theorem 2.6. Therefore, the above discussion in combination with (3.20) leads to

$$
\left\|\Phi_{1}\left(\cdot, y_{j}\right)\right\|_{H^{1}\left(\Omega_{0}\right)}-\left\|\widetilde{p}_{j}^{s}\right\|_{H^{1}\left(\Omega_{0}\right)} \leq\left\|\widetilde{p}_{j}\right\|_{H^{1}\left(\Omega_{0}\right)}=\left\|v_{j}\right\|_{H^{1}\left(\Omega_{0}\right)} \leq C \quad \text { uniformly for all } j \in \mathbb{N} \text {. }
$$

However, this is a contradiction since $\left\|\widetilde{p}_{j}^{s}\right\|_{H^{1}\left(\Omega_{0}\right)}$ is uniformly bounded and $\left\|\Phi_{1}\left(\cdot, y_{j}\right)\right\|_{H^{1}\left(\Omega_{0}\right)} \rightarrow \infty$ as $j \rightarrow \infty$. Then we have $\Gamma_{0}=\widetilde{\Gamma}_{0}$. This completes the proof of the theorem.

Remark 3.2 Theorem 3.1 can be easily extended to the two-dimensional case.

\section{Acknowledgements}

Not applicable.

\section{Funding}

This work was supported by the NNSF of China under grants 11871416, 11971273 and by the projects ZR2019MA027, ZR2018MA004 and ZR2017MA044 supported by Shandong Provincial Natural Science Foundation.

\section{Availability of data and materials}

Not applicable.

\section{Competing interests}

The authors declare that they have no competing interests

\section{Authors' contributions}

All authors contributed equally to the writing of this paper. All authors read and approved the final manuscript.

\section{Author details}

${ }^{1}$ School of Mathematics and Information Sciences, Yantai University, Yantai, P.R. China. ${ }^{2}$ School of Mathematics and Information Science, Shandong Technology and Business University, Yantai, P.R. China.

\section{Publisher's Note}

Springer Nature remains neutral with regard to jurisdictional claims in published maps and institutional affiliations.

Received: 14 February 2020 Accepted: 11 May 2020 Published online: 24 May 2020

\section{References}

1. Bao, G., Gao, Y., Li, P.: Time-domain analysis of an acoustic-elastic interaction problem. Arch. Ration. Mech. Anal. 229, 835-884 (2018)

2. Cakoni, F., Colton, D.: A uniqueness theorem for an inverse electromagnetic scattering problem in inhomogeneous anisotropic media. Proc. Edinb. Math. Soc. 46, 293-314 (2003)

3. Cakoni, F., Gintides, D., Haddar, H.: The existence of an infinite discrete set of transmission eigenvalues. SIAM J. Math. Anal 42, 237-255 (2010)

4. Colton, D., Kress, R.: Inverse Acoustic and Electromagnetic Scattering Theory, 2nd edn. Springer, Berlin (1998)

5. Colton, D., Kress, R., Monk, P.: Inverse scattering from an orthotropic medium. J. Comput. Appl. Math. 81, 269-298 (2007)

6. Elschner, J., Hsiao, G.C., Rathsfeld, A.: An inverse problem for fluid-solid interaction. Inverse Probl. Imaging 2, 83-120 (2008)

7. Elschner, J., Hsiao, G.C., Rathsfeld, A.: An optimization method in inverse acoustic scattering by an elastic obstacle. SIAM J. Appl. Math. 70, 168-187 (2009)

8. Elschner, J., Hu, G.: Uniqueness in inverse transmission scattering problems for multilayered obstacles. Inverse Probl. Imaging 5, 793-813 (2011)

9. Elschner, J., Hu, G.: Corners and edges always scatter. Inverse Probl. 31, Article ID 015003 (2015)

10. Gao, Y., Li, P., Zhang, B.: Analysis of transient acoustic-elastic interaction in an unbounded structure. SIAM J. Math. Anal. 49, 3951-3972 (2017)

11. Gilbarg, D., Trudinger, N.S.: Elliptic Partial Differential Equations of Second Order, 2nd edn. Springer, New York (1983)

12. Hähner, P.: On the uniqueness of the shape of a penetrable anisotropic obstacle. J. Comput. Appl. Math. 116, 167-180 (2000)

13. Hsiao, G.C., Kleinman, R.E., Roach, G.F.: Weak solutions of fluid-solid interaction problems. Math. Nachr. 218, 139-163 (2000) 
14. Hu, G., Salo, M., Vesalainen, E.V.: Shape identification in inverse medium scattering problems with a singular far-field pattern. SIAM J. Math. Anal. 48, 152-165 (2016)

15. Hu, G., Yang, J., Zhang, B., Zhang, H.: Near-field imaging of scattering obstacles via the factorization method. Inverse Probl. 30, Article ID 095005 (2014)

16. Isakov, V:: On uniqueness in the inverse transmission scattering problem. Commun. Partial Differ. Equ. 15, 1565-1587 (1990)

17. Jones, D.S.: Low-frequency scattering in elasticity. Q. J. Mech. Appl. Math. 34, 431-451 (1981)

18. Kar, M., Lin, Y.-H., Sini, M.: The enclosure method for the anisotropic Maxwell's system. SIAM J. Math. Anal. 47, 3488-3527 (2015)

19. Kar, M., Sini, M.: Reconstruction of interfaces from the elastic farfield measurements using CGO solutions. SIAM J. Math. Anal. 46, 2650-2691 (2014)

20. Kimeswenger, A., Steinbach, O., Unger, G.: Coupled finite and boundary element methods for fluid-solid interaction eigenvalue problems. SIAM J. Numer. Anal. 52, 2400-2414 (2014)

21. Kirsch, A., Ruiz, A.: The factorization method for an inverse fluid-solid interaction scattering problem. Inverse Probl. Imaging 6, 681-695 (2012)

22. Liu, H., Zhao, H., Zou, C.: Determining scattering support of anisotropic acoustic mediums and obstacles. Commun. Math. Sci. 13(4), 987-1000 (2015)

23. Liu, X., Zhang, B.: Direct and inverse obstacle scattering problems in a piecewise homogeneous medium. SIAM J. Appl. Math. 70, 3105-3120 (2010)

24. Monk, P., Selgas, V:: An inverse fluid-solid interaction problem. Inverse Probl. Imaging 3, 173-198 (2009)

25. Monk, P., Selgas, V.: Near field sampling type methods for the inverse fluid-solid interaction problem. Inverse Probl. Imaging 5, 465-483 (2011)

26. Nagayasu, S., Uhlmann, G., Wang, J.N.: Reconstruction of penetrable obstacles in acoustic scattering. SIAM J. Math. Anal. 43, 189-211 (2011)

27. Natroshvili, D., Kharibegashvili, S., Tediashvili, Z:: Direct and inverse fluid structure interaction problems. Rend. Mat. Appl. (7) 20, 57-92 (2000)

28. Potthast, R.: A point-source method for inverse acoustic and electromagnetic obstacle scattering problems. IMA J. Appl. Math. 61, 119-140 (1998)

29. Potthast, R.: On the convergence of a new Newton-type method in inverse scattering. Inverse Probl. 17, 1419-1434 (2001)

30. Qu, F., Yang, J.: On recovery of an inhomogeneous cavity in inverse acoustic scattering. Inverse Probl. Imaging 12 281-291 (2018)

31. Qu, F., Yang, J., Zhang, B.: An approximate factorization method for inverse medium scattering with unknown buried objects. Inverse Probl. 33, Article ID 035007 (2017)

32. Qu, F., Yang, J., Zhang, B.: Recovering an elastic obstacle containing embedded objects by the acoustic far-field measurements. Inverse Probl. 34, Article ID 015002 (2018)

33. Sanchez Hubert, J., Sánchez-Palencia, E.: Vibration and Coupling of Continuous Systems. Springer, Berlin (1989)

34. Yang, J., Zhang, B.: An inverse transmission scattering problem for periodic media. Inverse Probl. 27, Article ID 125010 (2011)

35. Yang, J., Zhang, B., Zhang, H.: The factorization method for reconstructing a penetrable obstacle with unknown buried objects. SIAM J. Appl. Math. 73, 617-635 (2013)

36. Yang, J., Zhang, B., Zhang, H.: Uniqueness in inverse acoustic and electromagnetic scattering by penetrable obstacles. J. Differ. Equ. 12, 6352-6383 (2018)

37. Yin, T., Hsiao, G.C., Xu, L.: Boundary integral equation methods for the two-dimensional fluid-solid interaction problem. SIAM J. Numer. Anal. 55, 2361-2393 (2017)

38. Yin, T., Hu, G., Xu, L., Zhang, B.: Near-field imaging of scattering obstacles with the factorization method II. Fluid-solid interaction. Inverse Probl. 32, Article ID 015003 (2016)

39. Yoshida, K.: Reconstruction of a penetrable obstacle by complex spherical waves. J. Math. Anal. Appl. 369, 645-657 (2010)

\section{Submit your manuscript to a SpringerOpen ${ }^{\circ}$ journal and benefit from:}

- Convenient online submission

- Rigorous peer review

- Open access: articles freely available online

- High visibility within the field

- Retaining the copyright to your article

Submit your next manuscript at $\gg$ springeropen.com 